\title{
Energy-Efficient Precoding for Multi-User Visible Light Communication with Confidential Messages
}

\author{
Son T. Duong*, Thanh V. Pham ${ }^{\dagger}$, Chuyen T. Nguyen*, and Anh T. Pham ${ }^{\dagger}$ \\ ${ }^{*}$ Hanoi University of Science and Technology, Vietnam. \\ ${ }^{\dagger}$ Computer Communications Lab., The University of Aizu, Japan. \\ Emails: *duongthanhson2808@gmail.com, *chuyen.nguyenthanh@hust.edu.vn, ${ }^{\dagger}\{$ tvpham, pham $\} @$ u-aizu.ac.jp
}

\begin{abstract}
In this paper, an energy-efficient precoding scheme is designed for multi-user visible light communication (VLC) systems in the context of physical layer security, where users' messages are kept mutually confidential. The design problem is shown to be non-convex fractional programming, therefore Dinkelbach algorithm and convex-concave procedure (CCCP) based on the first-order Taylor approximation are utilized to tackle the problem. Numerical results are performed to show the convergence behaviors and the performance of the proposed solution for different parameter settings.
\end{abstract}

Index Terms-Multi-user VLC, energy efficiency, physical layer security, precoding.

\section{INTRODUCTION}

Visible light communication (VLC) has been becoming an attractive wireless solution to complement existing technologies due to its high-capacity data transmission with licensefree spectrum. Aside from this, the technology also takes advantage of the widespread deployment of light-emitting diodes (LEDs). This naturally enables VLC to fit into the future ubiquitous networks.

However, multiple challenges still remain to make VLC more viable, among which security in terms of information privacy and confidentiality (especially in public areas) are of the most important issues [1]. In this respect, physical layer security (PLS) has emerged as novel paradigm to enhance secure communication by exploiting the randomness of the wireless channels, noise, and interference. The most promising implication of PLS is that a perfect secured communication can be achieved from information theoretic point of view. The secrecy of PLS is quantized by the secrecy rate that defines the maximum transmission rate at which unauthorized users are unable to extract any information from the received signals regardless of their computational capability. While PLS has been a well investigated topic in radio frequency (RF) communications, it has only been receiving considerable attention in the past few years in the case of VLC. In practical VLC systems, multiple LED luminaries should usually be deployed to provide a sufficient illumination. As such, multiple-input single-output (MISO) channels are more prevalent. In such scenarios, the degrees of freedom introduced by multiple LED transmitters enables the use of precoding as a means of secrecy enhancement [2]-[9].

Aside from the security, energy efficiency is another essential criterion in designing a communications system. This increasing attention to energy consumption comes from the current global effort to reduce the carbon footprint. In regard to PLS in VLC, there has been a few studies dealing with precoding design from the energy consumption perspective. In the case of one legitimate user and multiple eavesdroppers, the authors in [4] and [10] considered the problem of minimizing the power of the information-bearing signal while ensuring a minimum achievable secrecy rate. With the same configuration, [11], [12] focused on designing artificial noise-aided precoding. The total power consumption of the informationbearing signal and the artificial noise is then minimized taking into account predefined thresholds on the signal-tointerference-plus-noise ratios (SINRs) of the legitimate user and eavesdroppers.

In general, the above mentioned works concerned with the issue of minimizing the consumed power given that a certain secrecy requirement is fulfilled. This design approach, however, is not necessarily optimal from the perspective of energy efficiency, which is defined as the number of bits that can be transmitted per Joule. In other words, it is the ratio of the achievable (secrecy) rate to the total power consumption. Considering that an energy-efficient precoding design for PLS in multi-user (MU) VLC systems has not been studied, the objective of this paper is to fill this gap. Particularly, we examine an MU-MISO VLC system where each user treats others as eavesdroppers, hence its intended message must be kept confidential. The energy efficiency in the context of PLS of such system is then formulated using a lower bound on the achievable secrecy sum-rate. Regarding the power consumption, in addition to the power consumed for the information-bearing signal and circuitry operations (e.g., as in radio frequency (RF) communications), the lighting function of VLC requires an additional power for illumination, which also impacts the achievable secrecy rate. The optimal precoding design problem to maximize the energy efficiency is shown to be non-convex fractional programming, which renders finding an exact solution difficult. Hence, our approach is to find a sub-optimal yet computational efficient solution by employing the Dinkelbach algorithm and convex-concave procedure (CCCP). Simulations under different system parameters are conducted to verify the efficiency of the proposed solution.

The rest of the paper is structured as follows. The system model together with a formulation of the energy efficiency are described in Section II. Section III focuses on solving the 
precoding design to maximize the energy efficiency. Numerical results and related discussions are given in Section IV. Finally, Section V concludes the paper.

Notation: $\mathbb{R}^{m \times n}$ denotes the $m \times n$ real-valued matrices. The uppercase bold symbols, e.g. M, denote matrices, while the lowercase ones such as $\mathbf{v}$ represent column vectors. The transpose of $\mathbf{M}$ is written as $\mathbf{M}^{T}$. The $k$-th column vector of $\mathbf{M}$ is denoted as $\mathbf{m}_{k}$, while its $(i, j)$-th element is written as $m_{i, j}$. Additionally, $|\cdot|_{1},\|\cdot\|$ and $|\cdot|$ are $L_{1}$ norm, the Euclidean norm and absolute value operator, respectively.

\section{SySTEM MODEL}

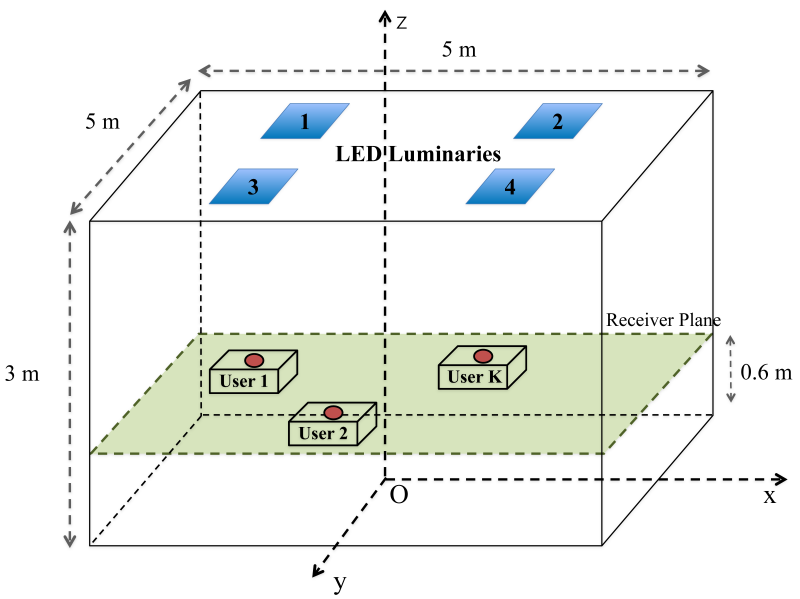

Fig. 1: A simple example of the considered MU-MISO VLC system with $N_{T}=4$ LED arrays and $K=3$ users.

Our considered MU-MISO VLC system as illustrated in Fig. 11 consists of $N_{T}$ LED luminaries and $K$ decentralized users, where each user is equipped with a photodiode (PD). It is reminded that the transmission is considered to be confidential if each user is not able to decode any information intended to the others.

\section{A. Signal Model}

Let $\mathbf{d}=\left[\begin{array}{llll}d_{1} & d_{2} & \cdots & d_{K}\end{array}\right]^{T} \in \mathbb{R}^{K \times 1}$ be the vector of data symbols for all users. Assume that the symbols are drawn from an $M$-ary pulse amplitude modulation ( $M$-PAM) and are modeled as a random variable (RV) $d$ following a certain distribution over $[-1,1]$ with zero-mean and variance $\sigma_{d}^{2}$. An information-bearing signal $s_{n}$ for the $n$-th LED transmitter is generated from a linear combination of the data vector and a precoder $\mathbf{v}_{n}=\left[\begin{array}{llll}w_{n, 1} & w_{n, 2} & \cdots & w_{n, K}\end{array}\right] \in \mathbb{R}^{1 \times K}$ as

$$
s_{n}=\mathbf{v}_{n} \mathbf{d} .
$$

For illumination, an DC bias $I_{n}^{\mathrm{DC}}$ should be added to $s_{n}$ to create a non-negative drive current $x_{n}$ for the LED. The drive current, in addition, needs to be constrained to a maximum threshold, i.e., $I_{\max }$, to ensure that LEDs operate normally. Therefore,

$$
0 \leq x_{n}=s_{n}+I_{n}^{\mathrm{DC}} \leq I_{\max }
$$

The emitted optical power of each LED luminary is given by

$$
P_{n}^{s}=\eta\left(s_{n}+I_{n}^{\mathrm{DC}}\right),
$$

where $\eta$ is the LED conversion factor. Denote $\mathbf{h}_{k}=$ $\left[h_{1, k} h_{2, k} \cdots h_{N_{T}, k}\right]^{T}$ is the $k$-th user's channel matrix, where $h_{n, k}$ is the line-of-sight (LoS) channel coefficient between the $n$-th LED array and the user. Details on the VLC channel model can be found in [1] and references therein. The electrical signal at the PD output is then given by

$$
\begin{aligned}
y_{k} & =\gamma \mathbf{h}_{k}^{T}\left[\begin{array}{llll}
P_{1}^{s} & P_{2}^{s} & \cdots & P_{N_{T}, k}^{s}
\end{array}\right]+n_{k} \\
& =\gamma \eta\left(\mathbf{h}_{k}^{T} \mathbf{w}_{k} d_{k}+\mathbf{h}_{k} \sum_{i=1, i \neq k}^{K} \mathbf{w}_{i} d_{i}+\mathbf{h}_{k} \mathbf{I}^{\mathrm{DC}}\right)+n_{k},
\end{aligned}
$$

where $\mathbf{w}_{\mathbf{k}}=\left[\begin{array}{llll}w_{1, k} & w_{2, k} & \cdots & w_{N_{T}, k}\end{array}\right]^{T}$ is the $k$-th user's precoder and $\mathbf{I}^{\mathrm{DC}}=\left[\begin{array}{llll}I_{1}^{\mathrm{DC}} & I_{2}^{\mathrm{DC}} & \cdots & I_{N_{T}}^{\mathrm{DC}}\end{array}\right]^{T}$. It is noted that since $\left|d_{i}\right| \leq 1$, we have

$$
-\left\|\mathbf{v}_{k}\right\|_{1} \leq s_{n} \leq\left\|\mathbf{v}_{k}\right\|_{1} .
$$

To ensure both (2) and (5), the following constraint should be satisfied

$$
\sum_{k=1}^{K}\left|w_{n, k}\right| \leq \min \left(I_{n}^{\mathrm{DC}}, I_{\max }-I_{n}^{\mathrm{DC}}\right) .
$$

The receiver noise $n_{k}$ in (4) can be modeled as a real-valued zero-mean Gaussian RV whose variance is given by

$$
\sigma_{k}^{2}=2 \gamma e \bar{P}_{k}^{r} B+4 \pi e A_{r} \gamma \chi_{\mathrm{amb}}(1-\cos (\Psi)) B+i_{\mathrm{amp}}^{2} B,
$$

where $\bar{P}_{k}^{r}=\eta \mathbf{h}_{k}^{T} \mathbf{I}^{\mathrm{DC}}$ is the average of received power at the $k$ th user, $A_{r}$ is the area of the PD, $e$ is the elementary charge, $\Psi$ is the optical field of view (FoV) ofthe PD, $B$ is the modulation bandwidth, $\chi_{\mathrm{amb}}$ is the ambient light photo-current, and $i_{\mathrm{amp}}$ is the pre-amplifier noise current density.

\section{B. Power Consumption}

We now analyze the total consumed power at LED transmitters, which can be expressed by

$$
P_{\text {total }}=P_{\mathrm{DC}}+P_{\mathrm{AC}},
$$

where $P_{\mathrm{DC}}$ and $P_{\mathrm{AC}}$ are the powers of the $\mathrm{DC}$ and $\mathrm{AC}$ currents, respectively. The DC current power includes the power used for illumination by LEDs denoted as $P_{\mathrm{DC}}$, LEDs and that used by other circuit components denoted as $P_{\mathrm{DC}}$, circuitry . While $P_{\mathrm{DC}}$, circuitry can be considered to be fixed, the power consumption of LEDs can be adjusted depending on the required dimming level. However, under a specific usage when the illumination level is not changed, it is reasonable to assume that $P_{\mathrm{DC}}$, LEDs is fixed as well. The DC power consumption is then given as

$$
P_{\mathrm{DC}}=P_{\mathrm{DC}, \mathrm{LEDs}}+P_{\mathrm{DC}, \text { circuitry }}
$$

with $P_{\mathrm{DC} \text {, LEDs }}$ being calculated as

$$
P_{\mathrm{DC}, \mathrm{LEDs}}=\sum_{n=1}^{N_{T}} U_{\mathrm{LED}} I_{n}^{\mathrm{DC}}
$$


where $U_{\text {LED }}$ being the forward voltage of the LEDs.

The AC currents comes from the output current (or voltage) from the precoders of LED drivers, therefore can be calculated as

$$
P_{\mathrm{AC}}=r \sum_{k=1}^{K} \sigma_{d}^{2}\left\|\mathbf{w}_{k}\right\|^{2},
$$

where $r$ is the equivalent resistance of the AC circuit. Without loss of generality, we denote $\xi=r \sigma_{d}^{2}$ as equivalent resistance, then rewrite the total power consumption as

$$
P_{\text {total }}=P_{\mathrm{DC}}+\xi \sum_{k=1}^{K}\left\|\mathbf{w}_{k}\right\|^{2} .
$$

\section{Energy Efficiency}

For demodulation, the DC component in the received signal in (4) is removed, yielding

$$
\bar{y}_{k}=\mathbf{h}_{k}^{T} \mathbf{w}_{k} d_{k}+\mathbf{h}_{k} \sum_{i=1, i \neq k}^{K} \mathbf{w}_{i} d_{i}+\bar{n}_{k},
$$

where $\bar{n}_{k}=\frac{n_{k}}{\gamma \eta}$. According to [7], a lower bound of achievable confidential secrecy rate of the $k-$ th user is given as

$$
\begin{aligned}
R_{s, k}(\mathbf{W})= & \frac{1}{2} \log _{2}\left(\frac{1+\sum_{i=1}^{K} a_{k}\left(\mathbf{h}_{k}^{T} \mathbf{w}_{i}\right)^{2}}{1+\sum_{i=1, i \neq k}^{K} b_{k}\left(\mathbf{h}_{k}^{T} \mathbf{w}_{i}\right)^{2}}\right) \\
& -\frac{1}{2} \log _{2}\left(1+\sum_{i=1, i \neq k}^{K} b_{i}\left(\mathbf{h}_{i}^{T} \mathbf{w}_{k}\right)^{2}\right),
\end{aligned}
$$

where $\mathbf{W}=\left[\begin{array}{llll}\mathbf{w}_{1} & \mathbf{w}_{2} & \cdots & \mathbf{w}_{K}\end{array}\right], a_{k}=\frac{\exp \left(2 h_{d}\right)}{2 \pi e \bar{\sigma}_{k}^{2}}$, and $b_{k}=\frac{\sigma_{d}^{2}}{\bar{\sigma}_{k}^{2}}$ with $\bar{\sigma}_{k}^{2}=\frac{\sigma_{k}^{2}}{(\gamma \eta)^{2}}$ and $h_{d}$ being the differential entropy of $d$. The energy efficiency with respect to the achievable secrecy sumrate of the considered system is therefore given by

$$
\Phi(\mathbf{W})=\frac{\sum_{k=1}^{K} R_{s, k}(\mathbf{W})}{P_{\mathrm{DC}}+\xi \operatorname{Tr}\left(\mathbf{W} \mathbf{W}^{T}\right)} .
$$

\section{Sub-optimal Precoding Design}

Our design objective is to maximize the energy efficiency while a minimum achievable secrecy rate for each user is guaranteed. Hence, the design problem can be formulated as follows

$$
\begin{array}{ll}
\max _{\mathbf{W}} & \Phi(\mathbf{W}), \\
\text { s.t. } & R_{s, k}(\mathbf{W}) \geq \lambda_{k}, \\
& \sum_{k=1}^{K}\left|w_{n, k}\right| \leq \min \left(I_{n}^{\mathrm{DC}}, I_{\max }-I_{n}^{\mathrm{DC}}\right),
\end{array}
$$

where $\lambda_{k}$ is the threshold for the secrecy rate of the $k$-th user. It is seen from (14) that the objective function is a non-concave fractional function with $\mathbf{W}$. This requires the use of Dinkelbach algorithm [13], which is efficient in solving fractional programming. Let $N(\mathbf{W})=\sum_{k=1}^{K} R_{s, k}(\mathbf{W}), D(\mathbf{W})=$ $P_{\mathrm{DC}}+\xi \operatorname{Tr}\left(\mathbf{W} \mathbf{W}^{T}\right)$. Then for some $\mu \geq 0$, the Dinkelbach algorithm involves solving the following

$$
\max _{\mathbf{W}} N(\mathbf{W})-\mu D(\mathbf{W}),
$$

$$
\begin{array}{ll}
\text { s.t. } & R_{s, k}(\mathbf{W}) \geq \lambda_{k}, \\
& \sum_{k=1}^{K}\left|w_{n, k}\right| \leq \min \left(I_{n}^{\mathrm{DC}}, I_{\max }-I_{n}^{\mathrm{DC}}\right) .
\end{array}
$$

to obtain a precoder $\mathbf{W}^{\prime}$. The optimal value $\mu^{*}=\frac{N\left(\mathbf{W}^{*}\right)}{D\left(\mathbf{W}^{*}\right)}$ is achieved when $N\left(\mathbf{W}^{\prime}\right)-\mu D\left(\mathbf{W}^{\prime}\right)=0$. This results in the Dinkelbach algorithm described as follows

\section{Algorithm 1: Dinkelbach-type algorithm for solving} (16).

Choose the maximum number of iterations $L_{\max , 1}$ and the error tolerance $\varepsilon_{1}$.

Initialize $\mu>0, l=0$.

while convergence $==$ False and $l \leq L_{\max , 1}$ do

$$
\begin{aligned}
& \text { For a given } \mu \text {, solve }(16) \text { to get } \mathbf{W}^{(l)} . \\
& \text { if } N\left(\mathbf{W}^{(l)}\right)-\mu D\left(\mathbf{W}^{(l)}\right) \leq \varepsilon_{1} \text { then } \\
& \begin{array}{l}
\text { convergence }==\text { True; } \\
\mathbf{W}^{*}=\mathbf{W}^{(l)} ; \\
\mu^{*}=\frac{N\left(\mathbf{W}^{(l)}\right)}{D\left(\mathbf{W}^{(l)}\right)} ;
\end{array} \\
& \text { else } \\
& \begin{array}{l}
\text { convergence }==\text { False } \\
l=l+1 ; \\
\text { Update } \mu=\frac{N\left(\mathbf{W}^{(l)}\right)}{D\left(\mathbf{W}^{(l)}\right)} ;
\end{array} \\
& \text { end }
\end{aligned}
$$$$
\text { end }
$$

Return the optimal $\mathbf{W}^{*}$ and $\mu^{*}$.

Nevertheless, it should be noted that (16) is not a convex optimization problem due to the non-concave objective function and the non-convex constraint in (16b). To overcome this problem, we make use of the CCCP approach based on the first-order Taylor approximation to approximate the original problem to a convex one. Specifically, we first introduce the following slack variables

$$
\begin{aligned}
& r_{1, k} \triangleq \frac{1}{2} \log _{2}\left(1+\sum_{i=1}^{K} a_{k}\left(\mathbf{h}_{k}^{T} \mathbf{w}_{i}\right)^{2}\right), \\
& p_{1, k} \triangleq \sum_{i=1}^{K} a_{k}\left(\mathbf{h}_{k}^{T} \mathbf{w}_{i}\right)^{2}, \\
& r_{2, k} \triangleq \frac{\Delta}{2} \log _{2}\left(1+\sum_{i=1, i \neq k}^{K} b_{k}\left(\mathbf{h}_{k}^{T} \mathbf{w}_{i}\right)^{2}\right), \\
& p_{2, k} \triangleq \sum_{i=1, i \neq k}^{K} b_{k}\left(\mathbf{h}_{k}^{T} \mathbf{w}_{i}\right)^{2}, \\
& r_{3, k} \triangleq \frac{1}{2} \log _{2}\left(1+\sum_{i=1, i \neq k}^{K} b_{i}\left(\mathbf{h}_{i}^{T} \mathbf{w}_{k}\right)^{2}\right), \\
& p_{3, k} \triangleq \sum_{i=1, i \neq k}^{K} b_{i}\left(\mathbf{h}_{i}^{T} \mathbf{w}_{k}\right)^{2},
\end{aligned}
$$


Then, the objective function $\sum_{k=1}^{K}\left(r_{1, k}-r_{2, k}-r_{3, k}\right)-$ $\mu\left(P_{\mathrm{DC}}+\xi \operatorname{Tr}\left(\mathbf{W} \mathbf{W}^{T}\right)\right)$ is a concave function with respect to $\mathbf{W}, r_{1, k}, r_{2, k}$, and $r_{3, k}$. Also, (16) can be rewritten as

$$
\max _{\substack{\mathbf{W}, r_{1, k}, r_{2, k}, r_{3, k} \\ p_{1, k}, p_{2, k}, p_{3, k}}} \sum_{k=1}^{K}\left(r_{1, k}-r_{2, k}-r_{3, k}\right)-\mu\left(P_{\mathrm{DC}}+\xi \operatorname{Tr}\left(\mathbf{W} \mathbf{W}^{T}\right)\right),
$$$$
\text { s.t. } r_{1, k} \leq \frac{1}{2} \log _{2}\left(1+p_{1, k}\right) \text {, }
$$$$
p_{1, k} \leq \sum_{i=1}^{K} a_{k}\left(\mathbf{h}_{k}^{T} \mathbf{w}_{i}\right)^{2}
$$$$
r_{2, k} \geq \frac{1}{2} \log _{2}\left(1+p_{2, k}\right),
$$$$
p_{2, k} \geq \sum_{i=1, i \neq k}^{K} b_{k}\left(\mathbf{h}_{k}^{T} \mathbf{w}_{i}\right)^{2}
$$$$
r_{3, k} \geq \frac{1}{2} \log _{2}\left(1+p_{3, k}\right) \text {, }
$$$$
p_{3, k} \geq \sum_{i=1, i \neq k}^{K} b_{i}\left(\mathbf{h}_{i}^{T} \mathbf{w}_{k}\right)^{2}
$$$$
r_{1, k}-r_{2, k}-r_{3, k} \geq \lambda_{k}
$$$$
\sum_{k=1}^{K}\left|w_{n, k}\right| \leq \min \left(I_{n}^{\mathrm{DC}}, I_{\max }-I_{n}^{\mathrm{DC}}\right) \text {. }
$$

It is seen that constraints $18 \mathrm{c}$, 18d, and 18f are still not convex. To cope with this, the first-order Taylor approximation is employed to approximate these non-convex constraints. The CCCP is then used to iteratively solve a sequence of approximating convex problems until a predefined convergence criterion is met [14]. Specifically, at the $m$-th iteration, the following problem is solved $\max _{\substack{\mathbf{W}, r_{1, k}, r_{2, k}, r_{3, k} \\ p_{1, k}, p_{2, k}, p_{3, k}}} \sum_{k=1}^{K}\left(r_{1, k}-r_{2, k}-r_{3, k}\right)-\mu\left(P_{\mathrm{DC}}+\xi \operatorname{Tr}\left(\mathbf{W W}^{T}\right)\right)$,

s.t.

$$
\begin{aligned}
p_{1, k} \leq & \sum_{i=1}^{K} a_{k}\left(\left(\mathbf{h}_{k}^{T} \mathbf{w}_{i}^{(m-1)}\right)^{2}\right. \\
& \left.+2\left(\mathbf{w}_{i}^{(m-1)}\right)^{T} \mathbf{h}_{k} \mathbf{h}_{k}^{T}\left(\mathbf{w}_{i}^{(m)}-\mathbf{w}_{i}^{(m-1)}\right)\right), \\
r_{2, k} \geq & \frac{1}{2} \log _{2}\left(1+p_{2, k}^{(m-1)}\right)+\frac{\left(p_{2, k}^{(m)}-p_{2, k}^{(m-1)}\right)}{2 \ln 2\left(1+p_{2, k}^{(m-1)}\right)}, \\
r_{3, k} \geq & \frac{1}{2} \log _{2}\left(1+p_{3, k}^{(m-1)}\right)+\frac{\left(p_{3, k}^{(m)}-p_{3, k}^{(m-1)}\right)}{2 \ln 2\left(1+p_{3, k}^{(m-1)}\right)},
\end{aligned}
$$

18b, 18e, 18g - 18i).

where $\mathbf{w}_{i}^{(m-1)}, p_{2, k}^{(m-1)}$ and $p_{3, k}^{(m-1)}$ are the solutions obtained from the previous iteration. Problem (19) is convex, thus can be solved efficiently by using standard optimization softwares , such as CVX [15]. Finally, (18) can be solved by the proposed Algorithm 2 using CCCP.

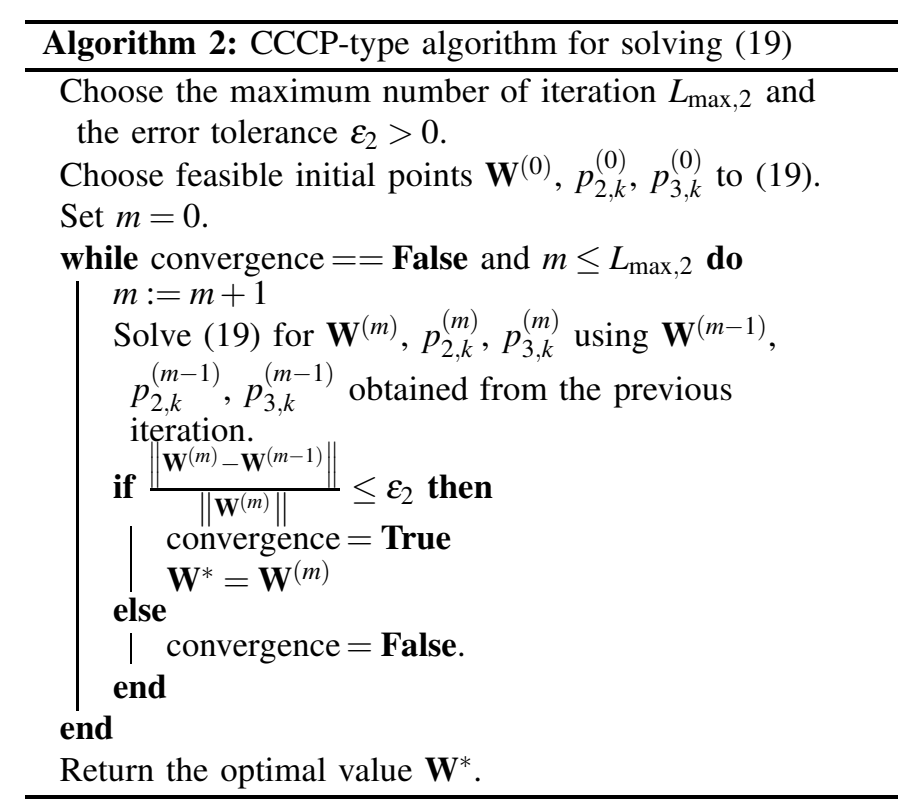

\section{Simulation Results And Discussions}

In this section, the performance convergence behaviors of the proposed solution are evaluated. Numerical results are obtained through averaging 10,000 different users' channel realizations. Unless otherwise noted, the following parameters are used for simulations. LED bandwidth $B=20 \mathrm{MHz}$, beam angle $\phi=120^{\circ}$, LED conversion factor $\eta=2$ W/A, active area of the PD $A_{r}=1 \mathrm{~cm}^{2}$, responsivity $\gamma=0.54$ A/W, FoV $\Psi=60^{\circ}$, optical filter gain $T_{s}(\psi)=1$, refractive index of the concentrator $\kappa=1.5$, ambient light photocurrent $\chi_{\mathrm{amp}}=10.93 \mathrm{~A} /\left(\mathrm{m}^{2} \cdot \mathrm{Sr}\right)$, and preamplifier noise current density, $i_{\mathrm{amp}}=5 \mathrm{pA} / \mathrm{Hz}^{-1 / 2}$. Moreover, the data symbols are assumed to be uniformly distributed over $[-1,1]$.

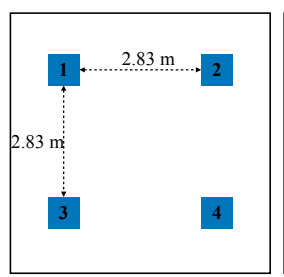

(a) $2 \times 2$ layout.

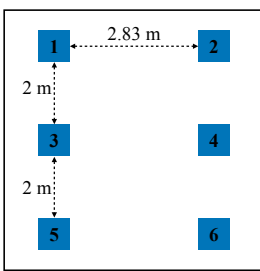

(b) $2 \times 3$ layout.

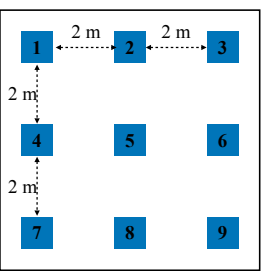

(c) $3 \times 3$ layout.
Fig. 2: Different layouts of LED transmitters.

Figure 3 illustrates the convergence of the proposed solution. For this purpose, three different scenarios of the numbers of LED transmitters and users, namely $\left(N_{T}, K\right)=(4,3),(6,4)$ and $(9,6)$, are taken into consideration. The configurations of LED transmitter are illustrated in Fig. 2 Additionally, the average emitted optical power of each LED luminary $\bar{P}_{n}^{s}=\eta I_{n}^{\mathrm{DC}}=30 \mathrm{dBm}, U_{\mathrm{LED}}=3.3 \mathrm{~V}, P_{\mathrm{DC} \text {, circuitry }}=8 \mathrm{~W}, \xi=3 \Omega$, and $\lambda_{k}$ 's $=0.5 \mathrm{bits} / \mathrm{s} / \mathrm{Hz}$ are set. Firstly, we examine the 
case that the initial precoder $\mathbf{W}^{(0)}$ in Algorithm 2 is chosen randomly. It is shown that considerably large numbers of iterations are required for the normalized energy efficiency to converge (i.e., 60, 75, and 90 iterations for $\left(N_{T}, K\right)=(4,3)$, $(6,4)$ and $(9,6)$, respectively). As we observe that the optimal solution is usually a near zero-forcing (ZF) precoder, one can choose the initial point as the $\mathrm{ZF}$ precoder to speed up the convergence. Indeed, simulation results revealed significant improvements in terms of the required number of iterations. Specifically, using ZF precoder as the initial point, roughly 8 , 9 , and 20 iterations are needed when $\left(N_{T}, K\right)=(4,3),(6,4)$ and $(9,6)$, respectively.

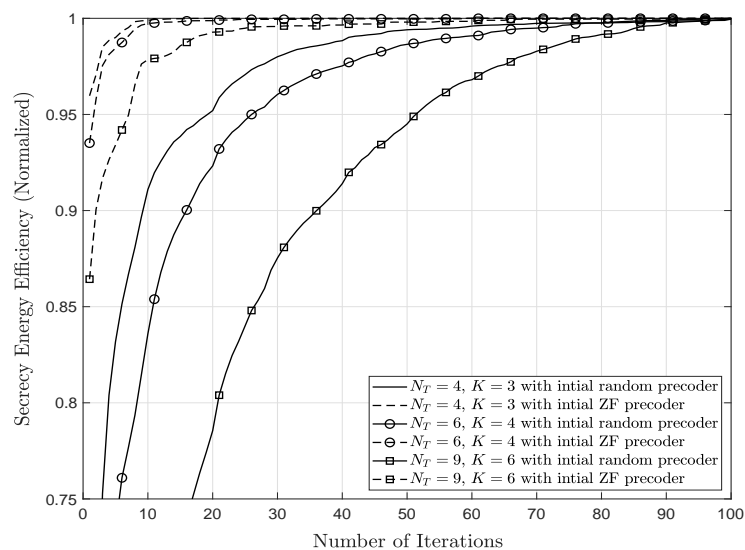

Fig. 3: Convergence behaviors of the proposed solution.

In Fig. 4, we assess the energy efficiency with respect to each luminary's average emitted optical power $\bar{P}_{n}^{s}$ for different values of circuitry power consumption where $\left(N_{T}, K\right)=(4,3)$. It is observed that the energy efficiency first increases with an increase in $\bar{P}_{n}^{s}$ until its maximum value. It then starts decreasing as $\bar{P}_{n}^{s}$ continues to increase. This phenomenon can be explained as follows. At its low value region, the increase in the achievable secrecy sum-rate due to increasing $\bar{P}_{n}^{s}$ is dominant in improving the energy efficiency. When $\bar{P}_{n}^{s}$ further increases, it becomes the dominant factor that reduces the energy efficiency as the achievable secrecy sum-rate only logarithmically increases with $\bar{P}_{n}^{s}$. We also notice that the optimal point of $\bar{P}_{n}^{s}$ increases in accordance with $P_{\mathrm{DC}}$, circuitry

\section{CONCLUSiOns}

In this paper, we have studied an energy-efficient precoding scheme for PLS in MU-MISO VLC systems. Due to the nonconvex nature of the design problem, Dinkelbach and CCCP algorithms were employed to find a sub-optimal solution with lower complexity. Numerical results shown that by choosing $\mathrm{ZF}$ precoders as the initial points for the CCCP algorithm, the rate of convergence of the proposed solution could be significantly improved. It was also demonstrated that there exist an optimal value of the average emitted optical power where the energy efficiency achieves its maximum value.

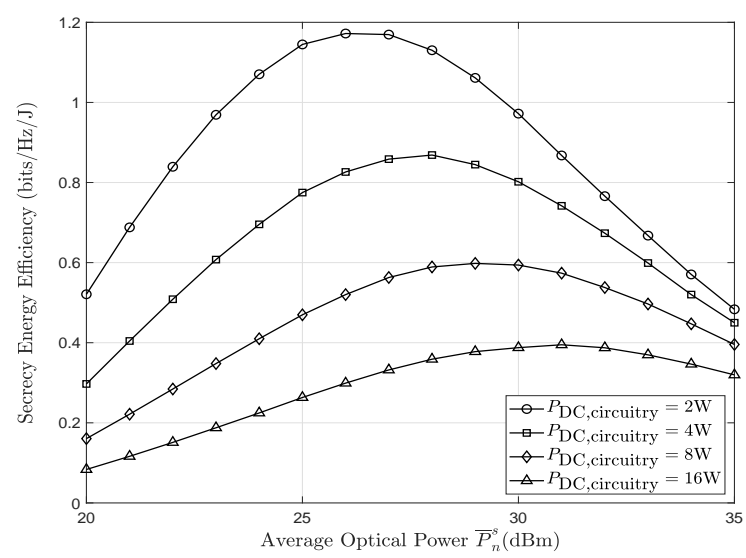

Fig. 4: Energy efficiency versus $\bar{P}_{n}^{s}$ with different $P_{\mathrm{DC}}$, circuitry.

\section{REFERENCES}

[1] M. A. Arfaoui et al., "Physical layer security for visible light communication systems: A survey," IEEE Commun. Surveys Tuts., vol. 22, no. 3, pp. 1887-1908, 2020.

[2] A. Mostafa and L. Lampe, "Physical-layer security for miso visible light communication channels," IEEE J. Sel. Areas Commun., vol. 33, no. 9, pp. 1806-1818, 2015.

[3] _ , "Optimal and robust beamforming for secure transmission in miso visible-light communication links," IEEE Trans. Signal Process., vol. 64, no. 24, pp. 6501-6516, 2016.

[4] S. Ma, Z.-L. Dong, H. Li, Z. Lu, and S. Li, "Optimal and robust secure beamformer for indoor miso visible light communication," J. Lightw. Technol., vol. 34, no. 21, pp. 4988-4998, 2016.

[5] T. V. Pham and A. T. Pham, "Secrecy sum-rate of multi-user miso visible light communication systems with confidential messages," Optik, vol. 151, pp. 65-76, 2017.

[6] M. A. Arfaoui, A. Ghrayeb, and C. Assi, "Achievable secrecy sum-rate of the miso vlc broadcast channel with confidential messages," in 2017 IEEE Global Communications Conference, 2017, pp. 1-6.

[7] M. A. Arfaoui, A. Ghrayeb, and C. M. Assi, "Secrecy performance of multi-user miso vlc broadcast channels with confidential messages," IEEE Trans. Wireless Commun., vol. 17, no. 11, pp. 7789-7800, 2018.

[8] S. Cho, G. Chen, and J. P. Coon, "Securing visible light communication systems by beamforming in the presence of randomly distributed eavesdroppers," IEEE Trans. Wireless Commun., vol. 17, no. 5, pp. 29182931, 2018.

[9] — , "Zero-forcing beamforming for active and passive eavesdropper mitigation in visible light communication systems," IEEE Trans. Inf. Forensics Secur., vol. 16, pp. 1495-1505, 2021.

[10] X. Liu et al., "Beamforming design for secure miso visible light communication networks with slipt," IEEE Transactions on Communications, vol. 68, no. 12, pp. 7795-7809, 2020.

[11] T. V. Pham and A. T. Pham, "Energy efficient artificial noise-aided precoding design for visible light communication systems," in $2020 \mathrm{In}$ ternational Conference on Computing, Networking and Communications (ICNC), 2020, pp. 507-512.

[12] — "Energy efficient artificial noise-aided precoding designs for secured visible light communication systems," IEEE Trans. Wireless Commun., vol. 20, no. 1, pp. 653-666, 2021.

[13] W. Dinkelbach, "On nonlinear fractional programming," Manag. Sci., vol. 13, no. 7, pp. 492-498, 1967.

[14] A. L. Yuille and A. Rangarajan, "The concave-convex procedure (cccp)," Neural Comput., vol. 15, no. 4, pp. 915-936, 2003.

[15] M. Grant and S. Boyd, "CVX: Matlab software for disciplined convex programming, version 2.1," http://cvxr.com/cvx. Mar. 2014.

\section{ACKNOWLEDGEMENT}

This work is supported by the Telecommunications Advancement Foundation (TAF) under Grant C-2020-2. 